\title{
Binational burden of American cutaneous leishmaniasis in Oiapoque, Amapá State, Brazil, bordering French Guiana
}

\author{
Thiago Vasconcelos-dos-Santos ${ }^{[1],[2],}$ Raimunda Cleide Gonçalves Chaves ${ }^{[3]}$, \\ Ghislaine Prévot ${ }^{[4]}$, Fernando Tobias Silveira ${ }^{[1]}$, Marinete Marins Póvoa ${ }^{[1],[2]}$ \\ and Elizabeth Ferreira Range[ ${ }^{[1],[5]}$
}

\author{
[1]. Programa de Pós-Graduação em Biologia de Agentes infecciosos e Parasitários, Instituto de Ciências Biológicas, \\ Universidade Federal do Pará, Belém, PA, Brasil. \\ [2]. Seção de Parasitologia, Instituto Evandro Chagas, Secretaria de Vigilância em Saúde, Ministério da Saúde, Ananindeua, PA, Brasil. \\ [3]. Coordenadoria de Vigilância em Saúde, Secretaria de Estado da Saúde, Macapá, AP, Brasil. \\ [4]. Département de Médecine, Ecosystèmes Amazoniens et Pathologie Tropicale, EA 3593, Labex CEBA, Université de Guyane, Cayenne, Guyane. \\ [5]. Laboratório Interdisciplinar de Vigilância Entomológica em Diptera e Hemiptera, Instituto Oswaldo Cruz, \\ Fundação Oswaldo Cruz, Rio de Janeiro, RJ, Brasil.
}

\begin{abstract}
Introduction: American cutaneous leishmaniasis (ACL) is regarded as a public health problem in the Oiapoque basin, between Brazil and French Guiana. Methods: Data on ACL occurrence/epidemiological profile and etiology were sourced from Brazilian health services and a reference laboratory. Rainfall correlation was also analyzed. Results: Majority of the ACL cases were observed in adult men working as gold miners. ACL incidence peaks appeared to be linked to periods 2 months after the dry season. Migratory flow was found to be a non-negligible complicating factor in epidemiological surveillance. Conclusions: Binational strategies are required to minimize exposure for high-risk populations.
\end{abstract}

Keywords: Leishmaniasis. Binational. Public health.

The Oiapoque basin is in the Guianan Ecoregion Complex, where the Oiapoque River borders Brazil and French Guiana (FG). On the Brazilian side, the region is bordered by the state of Amapá, where the municipality of Oiapoque is the primary socioeconomic connection to the FG villages (Figure 1). In that region, American cutaneous leishmaniasis (ACL) is endemic ${ }^{1}$ and is caused by at least three Leishmania species: L. (Viannia) guyanensis (81\%), L. (V.) braziliensis (17\%), and L. (V.) lainsoni $(2 \%)^{2}$. In this study, we aimed to present several epidemiological aspects of ACL in Oiapoque and its traits as a binational burden.

Primary data were sourced directly from epidemiological records located in the public health services offices (Brazilian side). Patients also sought ACL diagnosis at the Ralph

Corresponding author: Thiago Vasconcelos dos Santos.

e-mail: thiagovasconcelos@iec.gov.br

Orcid: 0000-0002-0229-4861

Received 18 June 2018

Accepted 20 September 2018
Lainson's Leishmaniasis Lab (Instituto Evandro Chagas [IEC], Ananindeua, Pará State, Brazil), where parasite isolation/characterization was performed according to previously established methodology ${ }^{3,4}$ (ethical approval CAAE: 57710416.2.0000.0019). Monthly average rainfall data were obtained from the Oiapoque Meteorological Station-A242 and ACL monthly/annual prevalence was obtained by searching the database of the Agency for Surveillance Coordination in Health of the Amapá State Health Secretary. The relationship between ACL occurrence and rainfall was evaluated with Pearson's correlation coefficient using the Past Software Package (Øyvind Hammer, Oslo, Norway), Version 3.22. The significance level was set at $5 \%$.

During 2008-2017, a total of 1,299 new ACL cases were documented by the health services in Oiapoque (an average of 118 cases/year), with 560 cases being demonstrated to be autochthonous for the municipality (average of 50 cases/year). An increasing number of ACL cases $(n=187)$ were observed in 2014; however, the greatest number of autochthonous cases was observed in $2012(\mathrm{n}=86)$. 


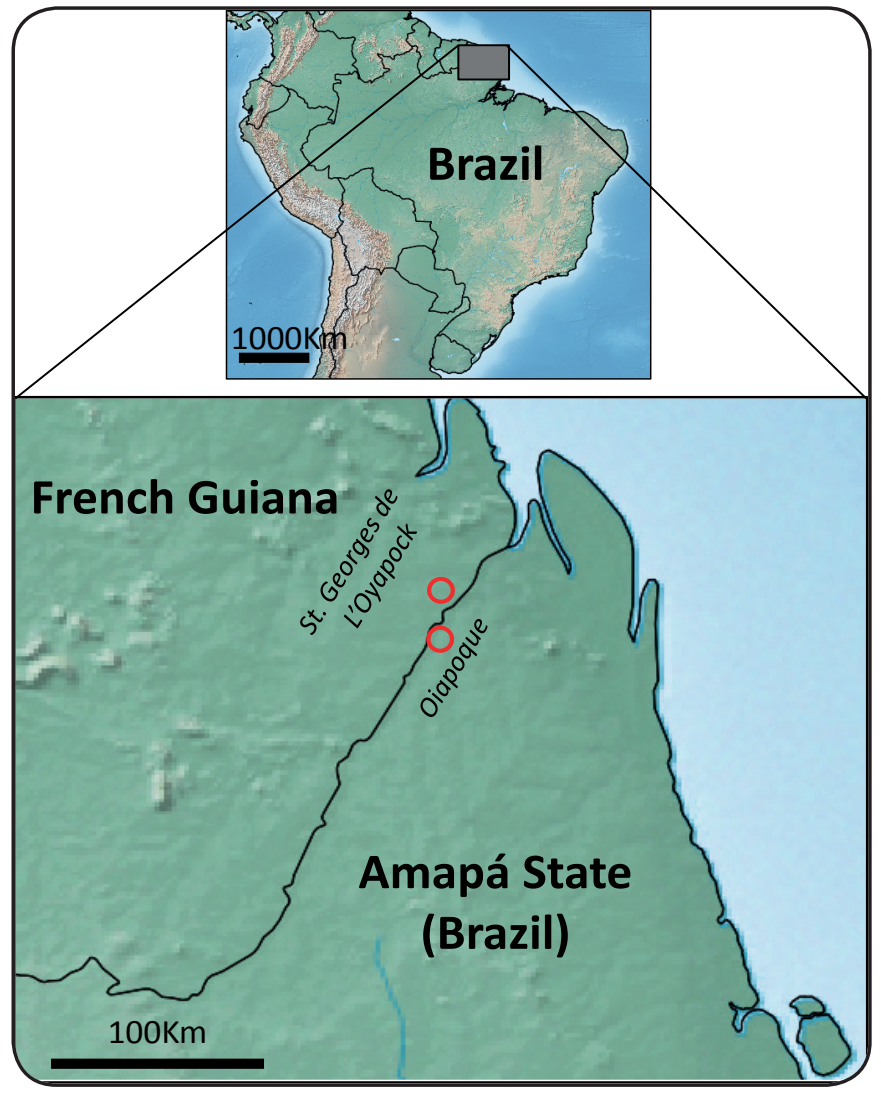

FIGURE 1: Map showing the border area of the Oiapoque basin, between the Brazilian State of Amapá and French Guiana, and the main closelyrelated villages/municipalities.

The frequency of ACL cases was greatest in December, January, February, March, and April, with these months accounting for $75 \%$ of the annual cases. Rainfall showed a moderate correlation with ACL occurrence $(r=0.646$; $p=0.0262$ ) (Figure 2). Climate is usually attributed as a nonnegligible explanatory factor for monthly ACL fluctuations, but strong correlations are not expected since there is a natural gap between transmission and diagnosis (incubation period ranging from 2 to 3 months) ${ }^{3}$. In FG, a decrease in rainfall is linked to an increase in ACL cases 2 months later ${ }^{5}$. Here, it was not possible to determine a relevant correlation, but graphical observations demonstrated a delay of 2 months between a dry period and ACL peaks. There is a reasonable explanation for this trend: In October, for instance, decreased rainfall can provide suitable conditions for forest activities and ecotourism, consequently raising the risk for human infection. The infected patients, therefore, seek a diagnosis after the appearance of lesions, 2 to 3 months later, coinciding with increased ACL statistics in January.

Due to the particular border characteristics of Oiapoque, ACL epidemiology is a mosaic of "binational" infections, reflecting in half of ACL notified cases being autochthonous from Brazil and the other half probably imported from French Guiana. The majority of the "imported" ACL cases are presumed to be from FG gold mines. Despite this finding, the proportion of autochthonous cases seems to be increasing slightly, as shown in the linear tendency in Figure 3. The rise can be attributed to a real increase in cases due to demographic growth or simply to the natural optimization of diagnostic/notification strategies in Oiapoque.

Thirty-five localities were declared as probable infection sites, most of them $(n=25)$ in the upper Oiapoque region. The localities with a concentration of ACL cases were the gold mines of Kuata (9.04\%), D21 (8.04\%), Anarry (6.28\%), and Sikini (5.52\%), all of which are located in FG territory. On the Brazilian side, ACL occurred in many localities, contributing to a dilution of frequencies, thus making it difficult to identify hotspots. Nevertheless, the gold mine of Cricou (2.51\%), and the village of Vila Brasil (2.26\%) stood out.

Gold miners comprised $49.14 \%$ of positive cases, followed by agriculturists (small farmers, $8.54 \%$ ) and students (7.26\%). Men (78.94\%) aged 21 to 50 years comprised the majority

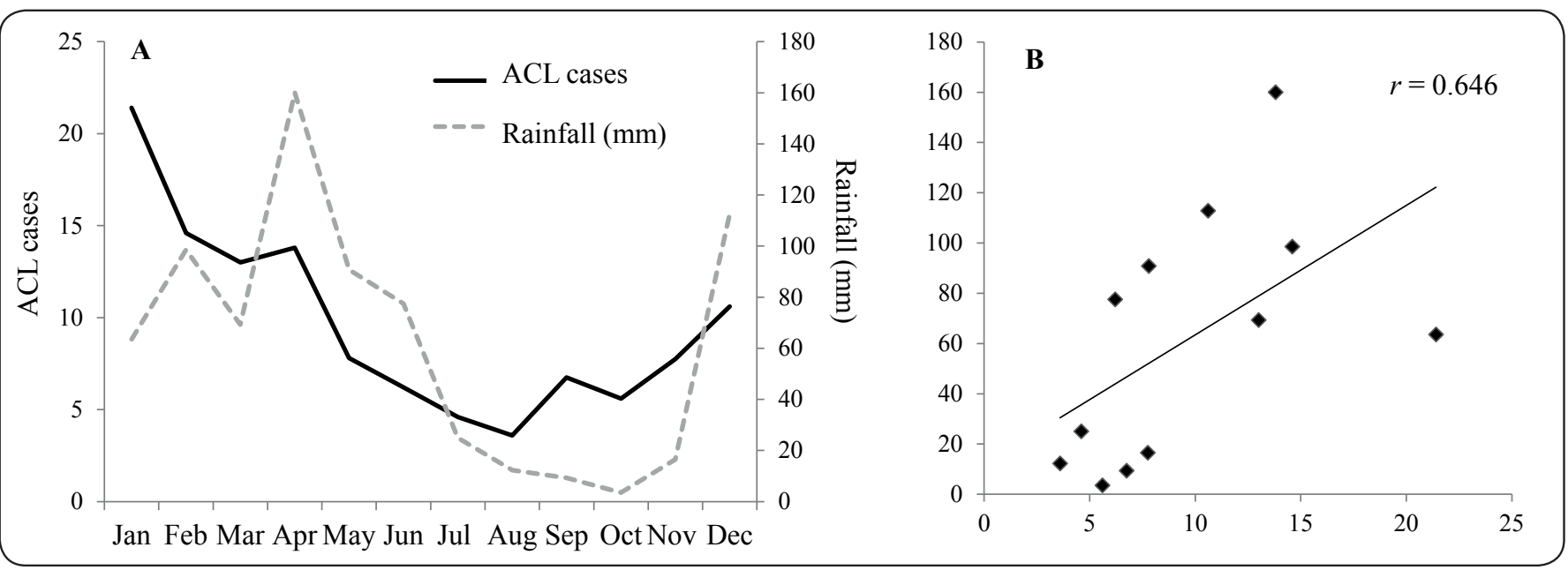

FIGURE 2: (A) Average monthly frequency of American cutaneous leishmaniasis cases and rainfall (B) with linear regression between ACL/rainfall fluctuations (2008-2017). 


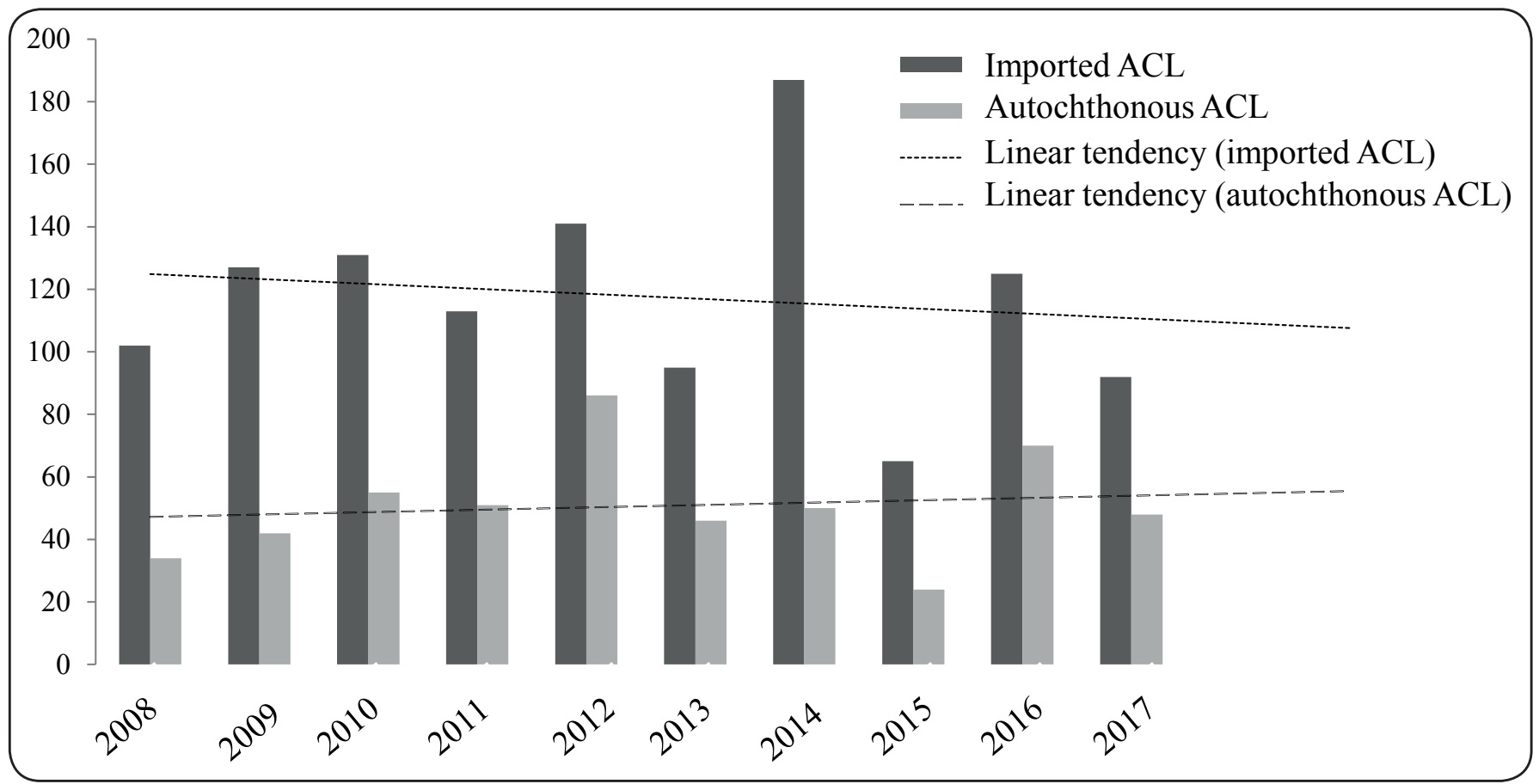

FIGURE 3: Annual fluctuation of autochthonous and imported American cutaneous leishmaniasis cases and linear tendency during a 10-year period (2008-2017).

of confirmed cases. In Saint Elie (FG), approximately 90\% of patients were illegal workers originating from the poorest northern Brazilian states ${ }^{6}$. Official data on the population of Oiapoque indicates 25,514 inhabitants in 2017. Unpredictable fluctuations in the illegal migrant population may be biasing the estimation of ACL incidence, but no consideration was given to this issue.

Occasional mucosal infections were reported in Oiapoque, mainly during the last 2 years, but Leishmania isolation/ characterization was not attempted. A supposed emergence of ACL due to $L$. (V.) braziliensis ${ }^{2,7,8}$ could explain this clinicalepidemiological profile. Alternatively, mucosal leishmaniasis due to $L$. $(V$.) guyanensis has been debated, and at least six cases have already been documented in $\mathrm{FG}^{9}$. Conversely, all Leishmania strains of ACL patients from Oiapoque attended in the IEC were isolated from cutaneous forms due to L. (V.) guyanensis (FT Silveira, personal observation) and the Oiapoque mucotropic parasite's identity remains speculative. Ecoepidemiology of $L$. (V.) braziliensis in the Oiapoque basin is poorly understood to date. Despite several records of human cases since the 1980s in $\mathrm{FG}^{2,7,8}$ and so far officially reported for Amapá State ${ }^{3}$, no phlebotomines or mammals were observed to be infected by that parasite in these two bordering territories/states. Putative vectors, Psychodopygus wellcomei, Ps. complexus, and Nyssomyia intermedia/Ny. neivai, have not been documented in the bordering region ${ }^{10}$. Even though Nyssomyia whitmani was reported, this fly is rarely present and there is strong evidence that this species is more likely to be associated with $L$. $(V$. $)$ guyanensis transmission in the Amazon/Guianan ACL scenario ${ }^{11}$.

Adult men exposed in the forest gold mines of the upper Oiapoque proved to be the majority of ACL patients treated by Brazilian health services, as has been suggested earlier. ACL incidence peaks seem to be linked to periods 2 months after the dry season. Information provided in the present communication may be useful for the establishment of preventive measures under binational responsibility. Surveillance strategies are needed, primarily to minimize exposure for high-risk populations, particularly in strategic areas, such as international borders.

Acknowledgments: The authors wish to thank the "Secretaria Municipal de Saúde de Oiapoque" team, particularly Andrew Breno Ferreira da Silva, Joessy de Cassia Folgado Aguiar, Carlos Luiz Amoras Sampaio, and Wanderson Pereira Pinto for their valuable support in ACL data collection.

Conflict of Interest: The authors declare that there is no conflict of interest.

Financial Support: This study was supported by IEC/SVS/MS and PROPESP/UFPA (01/2018).

\section{REFERENCES}

1. Rotureau B. Ecology of the Leishmania species in the Guianan Ecoregion Complex. Am J Trop Med Hyg. 2006;74(1):81-96.

2. Simon S, Nacher M, Carme B, Basurko C, Roger A, Adenis A, Ginouvès $M$, Demar $M$, Couppié P. Cutaneous leishmaniasis in French Guiana: revising epidemiology with PCR-RFLP. Trop Med Health. 2017;45:5.

3. Brasil- Ministry of Health. Secretary of Surveillance in Health. Department of Surveillance in Transmissible Diseases. Guide to surveillance of tegumentary leishmaniasis [in Portuguese]. 2nd ed. Brasília: Ministério da Saúde press; 2017, 189p. 
4. Simon S, Veron V, Carme B. Leishmania spp. identification by polymerase chain reaction-restriction fragment length polymorphism analysis and its applications in French Guiana. Diagn Microbiol Infect Dis. 2010;66(2):175-80.

5. Roger A, Nacher M, Hanf M, Drogoul AS, Adenis A, Basurko C, et al. Climate and leishmaniasis in French Guiana. Am J Trop Med Hyg. 2013;89(3):564-9.

6. Rotureau B, Joubert M, Clyti E, Djossou F, Carme B. Leishmaniasis among Gold Miners, French Guiana. Emerg Infect Dis. 2006;12(7):1169-70.

7. Martin-Blondel G, Iriart X, El Baidouri F, Simon S, Mills D, Demar M, et al. Outbreak of Leishmania braziliensis Cutaneous Leishmaniasis, Saül, French Guiana. Emerg Infect Dis. 2015;21(5):892-4.

8. Christen JR, Bourreau E, Demar M, Lightburn E, Couppié P, Ginouvès $M$, et al. Use of the intramuscular route to administer pentamidine isethionate in Leishmania guyanensis cutaneous leishmaniasis increases the risk of treatment failure. Travel Med Infect Dis. 2018; pii: S1477-8939(18)30040-1

9. Darrigade AS, Sambourg E, Duvignaud A, Sainte-Marie D, Labbé $\mathrm{S}$, Renaux A, Simon S, et al. Leishmanioses cutanéomuqueuses en Guyane: prédominance de l'espèce Leishmania guyanensis. Ann Dermatol Vener. 2014;141(12) Suppl:S29.

10. Vasconcelos dos Santos T, Prévot G, Ginouvès M, Duarte R, Silveira FT, Póvoa MM, et al. Ecological aspects of Phlebotomines (Diptera: Psychodidae) and the transmission of American cutaneous leishmaniasis agents in an Amazonian/ Guianan bordering area. Parasit Vectors. 2018;11(1):612.

11. Souza AAA, Barata IR, Silva MGS, Lima JAN, Jennings YLL, Ishikawa EAY, et al. Natural Leishmania (Viannia) infections of phlebotomines (Diptera: Psychodidae) indicate classical and alternative transmission cycles of American cutaneous leishmaniasis in the Guiana Shield, Brazil. Parasite. 2017;24:13. 